\title{
Graduate Medical Education on the Frontlines during the COVID-19 Pandemic in New York City - A Response to Promote Well-being
}

\author{
Saadia Akhtar, MD ${ }^{1 *}$, Sakshi Dua, MD¹, Paul J. Rosenfield, MD¹, Frank H. Basloe' ${ }^{1}$, Muhammad Khalid², \\ Jonathan A. Ripp, MD, MPH ${ }^{1}$, Michael Leitman, MD $^{1}$ \\ DOI: $10.18297 /$ jwellness/vol3/iss $2 / 9$ \\ Website: https://ir.library.louisville.edu/jwellness/

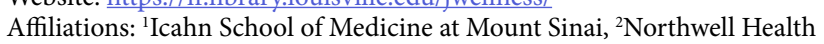 \\ Recommended Citation: Akhtar, Saadia; Dua, Sakshi; Rosenfield, Paul J.; Basloe, Frank H.; Khalid, Muhammad; Ripp, \\ Jonathan A.; Leitman, Michael (2021) "Graduate Medical Education on the Frontlines during the COVID-19 Pandemic \\ in New York City - A Response to Promote Well-being," Journal of Wellness: Vol. 3 : Iss. 2, Article 9. \\ Received Date: Dec 9, 2020 \\ Revised Date: May 192021 \\ Accepted Date: Sept 2, 2021 \\ Publication Date: Oct 12, 2021 \\ (c) (i)
}

\section{ABSTRACT}

Introduction: The COVID-19 pandemic has driven many health care institutions in the United States beyond their capacity. Physicians-in-training in graduate medical education programs have suffered the strain of providing patient care during this unprecedented time of crisis. The significant prevalence of pre-existing resident and fellow burnout and depression makes the need for action by institutions to support the well-being of residents and fellows even more urgent. We aim to describe innovative adaptations our Office of Graduate Medical Education implemented with the support of institutional leadership as responses to promote the well-being of residents and fellows on the frontlines during the COVID-19 pandemic.

Methods: The Office of Graduate Medical Education (GME), in collaboration with the Office of Well-being and Resilience, developed a set of resources and interventions to support trainees during the pandemic based on four major categories: workplace culture, personal factors and health, mental health support, and workplace efficiency and function. Examination of the capacity of existing services and gaps that needed to be filled in the rapidly evolving early days of the COVID pandemic led to a robust growth in resources. For example, the already established Student and Trainee Mental Health program was able to expand and adapt its role to serve trainee needs more effectively.

Results: We expanded resources to target trainee well-being across a broad array of domains within a short time frame. With investment in access to the Student and Trainee Mental Health program, utilization increased by $25.7 \%$, with 1,231 more visits in 2020 compared to the number of visits in 2019, prior to the COVID-19 pandemic. The creation of Recharge Rooms had a positive impact on the well-being of health care workers. After a single fifteen-minute experience in the Recharge Room, an average 59.6\% reduction in self-reported stress levels was noted by users. Other interventions were noted to be helpful in regular town hall meetings with trainees.

Conclusion: Addressing trainee well-being is an essential aspect of a crisis response. The Mount Sinai Health System was able to care for the physical, mental, psychosocial, and safety needs of our trainees thanks to the collaborative effort of a pre-existing institutional well-being program and the GME Office. The ability to implement such a response was enabled by our well-being foundation, which allowed leadership at the highest institutional level and the Office of GME to provide support in response to this unprecedented crisis.

\section{INTRODUCTION}

\section{Need for Innovation}

The COVID-19 pandemic pushed many hospital systems beyond their capacities. Health care workers, including physicians-in-training, were lauded as heroes but also suffered the strain of providing care to a large volume of critically ill patients, many of whom died from COVID-19. Concurrently, these residents and fellows dealt with the fears about whether they themselves might become seriously ill and possibly infect their families, the specter of the possible rationing of limited life-sustaining resources, the uncertainty of access to personal protective equipment (PPE), and the impact of isolation from family and friends [1].

${ }^{*}$ Correspondence To: Saadia Akhtar Email: Saadia.Akhtar@mountsinai.org
While this pandemic has been in many ways unprecedented for health care workers, negative outcomes such as burnout, depression, anxiety, post-traumatic stress disorder (PTSD), substance misuse and even suicide have been well documented following previous crises [2]. Early studies on health care workers exposed to COVID-19 have demonstrated depression, anxiety, insomnia, and distress, especially among women and frontline health care clinicians [3]. Given that this pandemic arrived in the context of a significant prevalence of pre-existing resident and fellow burnout and depression [4], we recognized the risk of adverse outcomes and the need for urgent action to support the well-being of our residents and fellows [5]. 
The first patient in New York City diagnosed with COVID-19 was evaluated at Mount Sinai Hospital on February 29, 2020. By April 9, 2020, the Mount Sinai Health System (MSHS) reached a peak of 1988 COVID-positive inpatients (out of 3,815 total beds), with 447 inpatients in intensive care units (ICU) on ventilators. The system mobilized the necessary resources to expand the bed capacity and redeploy health care workers from most specialties, including trainees, to COVID-dedicated units. On March 25, 2020, our institution was granted a Pandemic Emergency Status by the Accreditation Council for Graduate Medical Education (ACGME). The purpose of the emergency status was to allow institutional and program requirements to be relaxed while facing substantial and sustained disruption of graduate medical education operations. Residents and fellows were still required to be adequately supervised by faculty and to maintain compliance with all ACGME duty hour limits.

\section{APPROACH}

\section{Objective of the Innovation}

As the COVID-19 pandemic emerged in New York City, we were able to leverage the pre-existing infrastructure offered by the Icahn School of Medicine at Mount Sinai's Office of Wellbeing and Resilience (OWBR), which was established in 2018, in combination with the office of the largest graduate medical education (GME) program in the country (with over 2500 residents and fellows) to support our trainees [6]. The mission of the OWBR is to drive change by promoting initiatives aimed at removing barriers to well-being and reconnecting individuals with the meaning of their work.

Table 1: MSHS Well-being and Mental Health Resources

\begin{tabular}{|c|c|c|}
\hline & Established Resources & $\begin{array}{l}\text { Focused Resources During COVID } \\
\text { Pandemic }\end{array}$ \\
\hline Workplace Culture & $\begin{array}{l}\text { - GME Resident and Fellow Well- } \\
\text { being Subcommittee } \\
\text { - GME Well-being Champion Program }\end{array}$ & $\begin{array}{l}\text { Daily Mount Sinai Health } \\
\text { System Email } \\
\text { Communications with } \\
\text { Information and Support* } \\
\text { - Weekly GME Town Halls for } \\
\text { Trainees and Faculty* } \\
\text { - Transition to Virtual } \\
\text { Educational Programming* }\end{array}$ \\
\hline $\begin{array}{l}\text { Personal Factors and } \\
\text { Health }\end{array}$ & $\begin{array}{l}\text { - } \text { Competitive Salary } \\
\text { - } \text { Educational Stipend } \\
\text { - } \text { Fitilitated Group Discussion } \\
\text { - } \text { Food Pantry } \\
\text { - Mindfulness Training } \\
\text { - Narrative Medicine Workshops } \\
\text { - } \text { Putrition Counseling } \\
\text { - Positive Psychology } \\
\text { - Safe Travel } \\
\text { - Self-Care } \\
\text { - Siritual Care }\end{array}$ & $\begin{array}{l}\text { - } \text { Fitness Sessions* } \\
\text { - Hazard Pay } \\
\text { - Healthy Meals } \\
\text { - } \text { Lodging } \\
\text { - Nutrition Counseling* } \\
\text { - Parenting Resources* } \\
\text { - } \text { Pesking Discounts } \\
\text { - Scrub Access } \\
\text { - Self-Care* } \\
\text { - Spiritual Care* } \\
\text { - Stress Management* } \\
\text { - Taxi Subsidies }\end{array}$ \\
\hline $\begin{array}{l}\text { Mental Health } \\
\text { Support }\end{array}$ & $\begin{array}{l}\text { - } \text { Mental Health } \\
\text { Destigmatization Campaign } \\
\text { - } \text { Mental Health } \\
\text { Education Modules } \\
\text { - } \text { Mental Health } \\
\text { - Self-Screening Program } \\
\text { - } \text { Mental Health } \\
\text { Steering Committee } \\
\text { - Student and Trainee Mental Health } \\
\text { program }\end{array}$ & $\begin{array}{ll}\text { - } & \text { Crissis Line } \\
\text { - } & \text { Facilitated Group } \\
& \text { Discussions* } \\
\text { - } & \text { Mental Health Liaisons for } \\
& \text { Clinical Units* } \\
\text { - } & \text { Psychological First Aid* } \\
\text { - } & \text { Telepsychiatry with Student } \\
& \text { and Trainee Mental Health* }\end{array}$ \\
\hline $\begin{array}{l}\text { Workplace Efficiency } \\
\text { and Function }\end{array}$ & $\begin{array}{l}\text { - GME Clinical Work Intensity } \\
\text { Matching Grant Program } \\
\text { - GME Expectations for the } \\
\text { Promotion of Well-being } \\
\text { - GME Wellness Days Policy }\end{array}$ & $\begin{array}{ll}- & \text { Access to PPE } \\
- & \text { PPE Coaches* } \\
\text { - } & \text { Reconfiguring Teams by } \\
& \text { Location* }\end{array}$ \\
\hline
\end{tabular}

*Low-cost resources
The main areas of focus for the OWBR are organized into four categories: workplace culture, personal factors and health, mental health support, and workplace efficiency and function. An array of responses to the pandemic was built upon the existing infrastructure and principles of the OWBR, active GME partnership, and a model developed by our Employee, Faculty, and Trainee Crisis Support Task Force, to address trainee well-being in the midst of this crisis [7].

\section{Development Process}

The OWBR has a track record of providing resident and fellow well-being support to reduce burnout and depression [8]. The OWBR (comprised of GME and health system leadership along with the Employee, Faculty, and Trainee Crisis Support Task Force) took an inventory of existing resources and identified gaps in resources needed during the crisis. As part of this process, a well-being response was crafted, including well-established resources such as self-care and the implementation of new resources such as telepsychiatry. Our health system leadership focused on providing financing for both medical resources and psychosocial support, many of which were cost-effective initiatives needed for trainees (Table 1). Hill et al describe Emory University School of Medicine's effort to support the well-being of faculty and trainees during the pandemic, which also built on a pre-existing Wellness Working Group and provided a multidisciplinary approach [9].

\section{Implementation}

\section{A. Basic Needs and Self-Care}

During the pandemic, the OWBR worked with the GME Office and other institution partners to ensure prioritization of basic needs of the trainees: food, shelter, transport, and personal safety. Regular email communications, GME-led town halls (both for trainees and program directors), and a newly created website with up-to-date information on our location's COVID response, were provided to help support the institution's community.

Open access to a pantry stocked with refrigerated meals, snacks and beverages was expanded. For trainees who were deployed to the various COVID ICUs and Emergency Departments (EDs), ready-made meals were delivered directly to them. Subsidized taxi services, free parking and expanded shuttle services between hospital sites were made widely available because of the risks of using public transportation. Free hotel accommodations offered by local city businesses were also circulated to minimize trainee exposure to cohabitants.

Personal protective equipment (PPE) supplies never reached critically low levels owing to system-wide procurement efforts. All trainees completed PPE donning-doffing training modules adapted from the Centers for Disease Control and Prevention website. Supervision policies were revised to allow for cross-specialty faculty supervision of trainees redeployed to unfamiliar floors and units. Duty hours were monitored to maintain strict compliance with all regulations. A hazard pay initiative to compensate all trainees was also instituted. Access to on-call rooms and scrub machines for trainees was prioritized. Self-care techniques, stress management strategies, fitness and nutritional counseling continued to be made available using virtual platforms as part of our well-established focused resources. 


\section{B. Mental Health and Psychosocial Support}

Prior to the pandemic, the ACGME and individual training programs recognized the need to address the mental health of trainees directly through policy and initiatives. Many local GME programs have increased the resources available to trainees to reduce burnout and treat mental health needs, and our GME had successfully advocated for a significant expansion of the Student and Trainee Mental Health program prior to the pandemic [4].

At Mount Sinai, psychosocial needs during the pandemic were identified and met by offering newly formed support groups, including spiritual care sessions, which were swiftly converted to virtual platforms. If trainees felt that they were in distress or needed mental health assessment or treatment, many resources were available: mental health liaisons (MHLs) and volunteer behavioral health clinicians trained to proactively check in with all frontline staff to provide psychological first aid and respond to questions / assist with referrals. A 24-hour crisis line dedicated specifically to trainees and an expansion of the Student and Trainee Mental Health service providing virtual individual visits and group psychotherapy addressing the current pandemic were also made available. In response to the psychosocial effects of COVID-19 on our health care workers, the system created and launched a new Center for Stress, Resilience, and Personal Growth to provide ongoing screening, treatment, referral and resilience-building resources to faculty, staff and trainees for the foreseeable future beyond the acute phase of the pandemic.

\section{Support for Frontline Workers}

In an effort to provide support to frontline workers directly in the workplace, a number of initiatives were developed to respond to the crisis. Relief and Well-being Centers were established at the multiple sites within the health care system to offer nourishment of both mind and body. These well-being centers were created to provide access to interactive Recharge Rooms, which provided immersive private spaces that included meditative visual elements, seating, lighting, sound, scent and music. After a single fifteen-minute experience in the Recharge Rooms, users completed a short survey about their experience. An average of $59.6 \%$ reduction in self-reported stress levels was noted by users [10]. MHLs were often present (e.g., in EDs, ICUs and inpatient floors) to provide immediate staff access to mental health support.

\section{DISCUSSION}

With an understanding that large-scale medical crises exact a toll on health care workers, causing high levels of stress, anxiety, depression and burnout, our healthcare system sought to proactively mitigate the adverse effects of the COVID-19 pandemic among trainees and other health care workers. We recognized the concerns about safety in the workplace, in commuting to and from work, and in potentially exposing oneself and loved ones to COVID. We acknowledged the intensity of frequent exposure to death, and the risk of emotional exhaustion, from internal provider feedback and the emerging literature on health care worker wellness in China $[1,11]$.
We prioritized the physical, mental, psychosocial, and safety needs of our trainees thanks to the collaborative effort of a pre-existing institutional well-being program and the GME Office. With a focused approach, we implemented a response that resulted in increased utilization of mental health resources and reduction in self-reported stress levels. The ability to carry out such a comprehensive response was enabled by our well-being foundation, which allowed leadership at the highest institutional level and the Office of GME to quickly provide support in response to this unprecedented crisis.

By using the pre-existing framework of the four foundational pillars of OWBR's mission as noted in Table 1, we were expeditiously able to meet trainees' needs as the pandemic rapidly unfolded. We were able to demonstrate increased utilization of both pre-existing and newly established resources related to the pandemic. The outcomes of this expansion of resources included the increased utilization of the Student and Trainee Mental Health program, which registered 1,231 more visits in 2020 compared to the number of visits in 2019 (prior to the pandemic), representing a $25.7 \%$ increase. Utilization of the Recharge Rooms resulted in positive outcomes. After a single fifteen-minute experience in the Recharge Room, an average $59.6 \%$ reduction in self-reported stress levels was noted by users [9].

While we already had an established trainee Well-being Champion Program to address issues related to workplace culture pre-pandemic, during our surge we quickly escalated the frequency of informational communications and virtual 'facetime' sessions with GME leadership. This provided to trainees and their champions useful and up-to-date information during the rapidly unfolding pandemic-related events. At the same time, we supported trainees' educational needs by switching from in-person education to an online platform. Innovative formats for both communication-related meetings and educational events included early adoption of technology using both institutional and personal accounts.

Cognizant of trainees' personal needs and pandemic-related fears, we prioritized access to adequate PPE, meals, transport and accommodation. Our pandemic operations included expanded meal pantry, scrub, child-care and ride sharing access. Workplace efficiency was intensified during the surge by reconfiguring team-based care and geographic deployment. We realized that while some of these extra measures required institutional and hospital-based resources, others were leveraged in partnership with local willing businesses such as hotels, restaurants and ride sharing companies. This unique institutional and private business sector partnership in the middle of an unfolding crisis was successful.

Measuring the effect of these rapidly adopted well-being resources was an important aspect of the well-being response and helped determine how to allocate finite resources for maximum impact. We maintained regular communication with trainees to obtain feedback and ensure concerns were being heard and addressed in real-time. We also understood that visibility of the leadership in solidarity with the trainees was as important as the implementation of enhanced well-being resources. The lessons learned by our health system have been invaluable. We hope this work can serve as a guide to other 
institutions dealing with crises and illustrate how pre-existing foundational resources can be readily leveraged to respond to emergencies and ensure trainee well-being.

\section{REFERENCES}

1. Shreffler J, Petrey J, Huecker M. The Impact of COVID-19 on Healthcare Worker Wellness: A Scoping Review. West J Emerg Med. 2020 Aug;21(5):1059-66.

2. DePierro J, Lowe S, Katz C. Lessons learned from 9/11: mental health perspectives on the COVID-19 pandemic. Psychiatry Res. 2020 Jun;288:113024.

3. Lai J, Ma S, Wang Y, et al. Factors Associated With Mental Health Outcomes Among Health Care Workers Exposed to Coronavirus Disease 2019. JAMA Netw Open. 2020;3(3):e203976. Published 2020 Mar 2. https://doi. org/10.1001/jamanetworkopen.2020.3976.

4. Ripp JA, Privitera MR, West CP, Leiter R, Logio L, Shapiro J, et al. Well-Being in Graduate Medical Education: A Call for Action. Acad Med. 2017 Jul;92(7):914-7.

5. Shah K, Chaudhari G, Kamrai D, Lail A, Patel RS. How Essential Is to Focus on Physician's Health and Burnout in Coronavirus (COVID-19) Pandemic?. Cureus. 2020;12(4):e7538. Published 2020 Apr 4. https://doi. org/10.7759/cureus.7538.

6. Accreditation Council for Graduate Medical Education (ACGME). List of Sponsoring Institutions by Resident/
Fellow Count. [Internet]. 2020. [cited 2020 Dec 1]. Available from: https://apps.acgme.org/ads/Public/Reports/ Report $/ 20$

7. Ripp J, Peccoralo L, Charney D. Attending to the Emotional Well-Being of the Health Care Workforce in a New York City Health System During the COVID-19 Pandemic [published online ahead of print, 2020 Apr 10]. Acad Med. 2020 Aug;95(8):1136-9.

8. Bui AH, Ripp JA, Oh KY, Basloe F, Hassan D, Akhtar S, et al. The impact of program-driven wellness initiatives on burnout and depression among surgical trainees. Am J Surg. 2020 Feb;219(2):316-21.

9. Hill CC, Gomes PG, Feng AH, et al. Perspective: Responding to the Well-Being of Health Care Workers and Learners in Academic Medicine During the COVID-19 Pandemic. J Wellness. 2020;2(1):1-2. Available from: https://ir.library. louisville.edu/jwellness/vol2/iss $1 / 8$

10. Putrino D, Ripp J, Herrera JE, Cortes M, Kellner C, Rizk D, et al. Multisensory, Nature-Inspired Recharge Rooms Yield Short-Term Reductions in Perceived Stress Among Frontline Healthcare Workers. Front Psychol. 2020 Nov;11:560833-560833.

11. Que J, Shi L, Deng J, Liu J, Zhang L, Wu S, et al. Psychological impact of the COVID-19 pandemic on healthcare workers: a cross-sectional study in China. Gen Psychiatr. 2020 Jun;33(3):e100259. 